\title{
Über die Einwirkung von alkoholischem Natron auf Isobutyraldehyd
}

\author{
von
}

\section{Dr. Adolf Franke.}

Aus dem chemischen Universitätslaboratorium des Hofrathes Adolf Lieben.

(Vorgelegt in der Sitzung am 22. October 1896.)

In den Bulletins de la société chimique, 13, p. 1048 hat Urbain eine Arbeit über die Einwirkung von alkoholischem Natron auf Isobutyraldehyd veröffentlicht. Die Ergebnisse derselben sind im Wesentlichen folgende:

I. Isobutyraldehyd liefert, mit $5 \%$ alkoholischem Natron ohne Kühlung behandelt, drei Producte, eine unter gewöhnlichem Drucke bei $140^{\circ} \mathrm{C}$. siedende, leicht bewegliche Flüssigkeit, das 2, 2, 4-Trimetylpenten-3, al-1, ein bei $125-130^{\circ} \mathrm{C}$. (14 $\mathrm{mm}$ ) siedendes dickes Öl, das 2,5-Dimethylhexan-3, 4-olon und einen krystallisirten Körper, der bei $180^{\circ} \mathrm{C} .(14 \mathrm{~mm})$ destillirt und nicht näher untersucht wurde.

II. Isobutyraldehyd liefert in sonst gleicher Weise, jedoch unter Kühlung behandelt, ein bei $95^{\circ} \mathrm{C}$. $(14 \mathrm{~mm})$ destillirendes Product von der Zusammensetzung $\mathrm{C}_{8} \mathrm{H}_{16} \mathrm{O}_{2}$ und der Constitution eines 2,2,4-Trimethylpentan-1-al-3-ol.

Wie aus der Originalabhandlung ersichtlich ist, legte Urbain seinen Betrachtungen die seither ${ }^{1}$ umgestossene Glycolformel Fossek's ${ }^{2}$ zu Grunde, weshalb seine Schlüsse von vorneherein irrig sein mussten. Dies sowohl, wie der Umstand, dass er bei der Einwirkung von alkoholischem Natron auf Isobutyraldehyd in der Wärme, im Gegensatz zu den

J Monatshefte für Chemie, 1896, S. 45.

2 Monatshefte für Chemie, 1883, S. 663. 
bisherigen Erfahrungen mit alkoholischem Kali (wobei man fast quantitativ Glycol und Isobuttersäure erhält), kein Glycol erhielt, veranlassten mich, die Versuche Urbain's zu wiederholen.

$\mathrm{Zu} 20 \mathrm{~g}$ reinen Isobutyraldehydes liess ich $20 \mathrm{~g} 5 \%$ alkoholischen Natrons tropfenweise zufliessen. Schon die ersten Tropfen bewirkten bedeutende Temperaturerhöhung, so dass ich, um das Kochen des Aldehydes zu verhindern, mit Wasser kühlen musste. Das weiter einfliessende alkoholische Natron bewirkte keine Temperaturerhöhung mehr. Nachdem der Geruch nach Aldehyd fast vollständig verschwunden und das schwach gelb gefärbte, dickflüssige Reactionsgemisch 12 Stunden stehen gelassen worden war, wurde der Alkohol am Wasserbade abdestillirt, der Rückstand mit Wasser gewaschen und der Destillation im Vacuum unterworfen. Bei $14 \mathrm{~mm}$ Druck ging von $120-122^{\circ} \mathrm{C}$. ein dickes farbloses Ö1 über, das in der Vorlage zu einem Krystallbrei erstarrte. Nach dem Abpressen zwischen Filtrirpapier zeigten die Krystalle den Schmelzpunkt $51^{\circ}$ C. und die das Glycol ${ }^{1}$ charakterisirende Form von monoklinen Tafein.

Von den drei Producten, die Urbain erhalten zu haben behauptet, erhielt ich keines, obwohl ich mich bei wiederholten Versuchen strenge an seine Vorschrift hielt.

Was den Punkt II der Urbain'schen Abhandlung betrifft, kam ich zu dem nämlichen Resultate wie er und bin in der Lage, die von Urbain vermuthete, aber aus unrichtigen Prämissen abgeleitete Formel mit Sicherheit festzustellen.

$\mathrm{Zu} 20 \mathrm{~g}$ reinen Isobutyraldehydes liess ich, wieder der Vorschrift Urbain's folgend, $20 \mathrm{~g} 5 \%$ alkoholischen Natrons bei $-20^{\circ} \mathrm{C}$. tropfenweise einfliessen. Die ersten Tropfen bewirkten starke Erwärmung (bis $\mathrm{zu}+5^{\circ} \mathrm{C}$.), und nur durch sehr langsames Zutropfen und sorgfältige Kühlung wurden höhere Temperaturen vermieden. Um weitere Einwirkung des Alkalis zu verhindern, wurde noch in der Kälte Kohlensäure eingeleitet, dann mit Wasser gewaschen und das dicke farblose Öl abgehoben. Der Versuch, das Product zu destilliren,

1 Monatshefte für Chemie, 1896, S. 37. 
scheiterte daran, dass es selbst im Vacuum nur unter vollständiger Zersetzung, d. i. Umwandlung in Aldehyd, übergeht.

Diese auffallende Eigenschaft sowohl, wie sein Geruch, Geschmack und seine Consistenz liessen die Identität mit jenem Polymeren des Isobutyraldehydes, d. i. das entsprechende Aldol vermuthen, welches Brauchbar durch Einwirkung von wässerigem Kali oder concentrirter Pottaschelösung in der Kälte erhalten hat. Diese Vermuthung wurde durch das chemische Verhalten des Körpers bestätigt.

\section{Oxydation.}

Sowohl in neutraler, wie in schwefelsaurer Lösung wurde das Product mit Kaliumpermanganat zusammengebracht und längere Zeit bei gewöhnlicher. Temperatur stehen gelassen. Nachdem Entfärbung eingetreten war, wurde dem Reactionsgemisch durch Äther eine fixe Säure entzogen, welche den Schmelzpunkt $92^{\circ}$ C. zeigte, also identisch ist mit jener Säure, die ich durch Oxydation des Glycols erhalten und als 2,2,4Trimethylpentan-3-olsäure ${ }^{1}$ erkannt habe. Auch auf dem Wege über das Oxim und Nitril ist es mir gelungen, zur selben Oxysäure zu kommen.

\section{Oximversuch.}

Mit der berechneten Menge Hydroxylaminchlorhydrat und Soda in weingeistiger Lösung zusammengebracht, lieferte der Körper ein zähflüssiges farbloses Product, welches bei $140^{\circ}$ $(16 \mathrm{~mm})$ destillirte.

\section{Stickstoffbestimmung.}

$0 \cdot 2143 \mathrm{~g}$ Substanz gaben $16.6 \mathrm{~cm}^{3}$ Stickstoff, bei $22^{\prime} \mathrm{C}$. und $742 \mathrm{~mm}$ Barometerstand.

Auf 100 Theile:

$$
\mathrm{N} \ldots \ldots \cdot 9 \cdot 05
$$

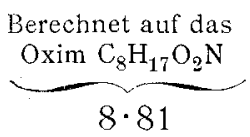

1 Monatshefte für Chemie, 1896, S. 44. 
Dieses Oxim des Aldols wurde durch neunstündiges Erhitzen mit Essigsäureanhydrid im Einschmelzrohr auf $120^{\circ} \mathrm{C}$. in das entsprechende Monoacetylderivat des Nitrils übergeführt, eine farblose, bei $112^{\circ} \mathrm{C}$. (circa $16 \mathrm{~mm}$ ) destillirende, schwach nach Blausäure riechende Flüssigkeit.

Analyse.

$0.2365 \mathrm{~g}$ Substanz gaben $0.5714 \mathrm{~g} \mathrm{CO}_{2}$ und $0.2010 \mathrm{~g} \mathrm{H}_{2} \mathrm{O}$. Auf 100 Theile:

\begin{tabular}{|c|c|}
\hline & $\begin{array}{l}\text { Berechnet auf } \\
\mathrm{C}_{10} \mathrm{H}_{17} \mathrm{O}_{2} \mathrm{~N}\end{array}$ \\
\hline $65 \cdot 89$ & $65 \cdot 57$ \\
\hline $\mathrm{H} \ldots \ldots$ & $9 \cdot 29$ \\
\hline
\end{tabular}

Durch Verseifung dieses Nitriles mit wässerigem Kali erhielt ich, wie schon oben erwähnt, die Oxysäure.

Durch diese Versuche ist die Constitution des Einwirkungsproductes von alkoholischem Natron in der Kälte festgestellt.

$$
\begin{array}{lll}
\mathrm{C}\left(\mathrm{CH}_{3}\right)_{2}-\mathrm{CHOH} . \mathrm{CH}\left(\mathrm{CH}_{3}\right)_{2} & \mathrm{C}\left(\mathrm{CH}_{3}\right)_{2}-\mathrm{CHOH} . \mathrm{CH}\left(\mathrm{CH}_{3}\right)_{2} \\
& 1 \\
\mathrm{CHO} & \mathrm{COOH} & \\
& &
\end{array}
$$

Es wäre noch auf die Reduction zu verweisen, welche Brauchbar ${ }^{1}$ mit Natriumamalgam in neutraler Lösung ausgeführt hat, und die das Glycol lieferte. Bei Behandlung des Aldols mit alkoholischem Natron oder Kali in der Wärme erhielt ich ebenfalls das Glycol neben Isobuttersäure, beides in nahezu der berechneten Menge.

Wenn daher Urbain am Schlusse seiner Arbeit in Bezug auf den Hergang der Reaction bei der Einwirkung von alkoholischem Kali auf Isobutyraldehyd (wobei man das Glycol $\mathrm{C}_{8} \mathrm{H}_{18} \mathrm{O}_{2}$, Isobuttersäure und ganz geringe Mengen der Oxysäure erhält) die Ansicht ausspricht, dass zunächst das Aldol zugleich mit einem isomeren Ketol entstehe; dass ferner bei der weiteren Einwirkung letzteres zum Glycol (dem er Fos se k's irrige Formel des "Diisopropylglycols « beilegt) reducirt und

1 Monatshefte für Chemie, October 1896. 
676 A. Franke, Einwirk. von alkohol. Natron auf Isobutyraldeh yd.

auf Kosten dieser Reduction ersteres zur Oxysäure, respective zu Isobuttersäure oxydirt werde; so sind wir jetzt in der Lage, diese Erklärung in folgender Weise richtig zu stellen:

Das Alkali, sei es wässerige Kalilauge oder gesättigte Pottaschelösung, oder endlich alkoholisches Natron oder Kali, wirkt zunächst condensirend auf den Isobutyraldehyd ein, und zwar im Sinne der Lieben-Zeisel'schen Regel:<smiles>CC(C)C=O</smiles><smiles>CC(C)C=O</smiles><smiles>CC(C)C(O)C(C)(C)C=O</smiles>

Dieses so entstandene Aldol bildet bei der weiteren Einwirkung von Alkali durch gleichzeitige Reduction und Oxydation das 2, 2, 4-Trimethylpentan-1,3-diol (Fossek's Diisopropylglycol) und Isobuttersäure. 